\title{
SIMULATING NAVIGATION WITH VIRTUAL 3D GEOVISUALIZATIONS - A FOCUS ON MEMORY RELATED FACTORS
}

\author{
I. Lokka*, A. Çöltekin \\ Department of Geography, University of Zurich, Zurich, Switzerland - (ismini-eleni.lokka, arzu.coltekin)@ geo.uzh.ch
}

ThS16: Perceptual and cognitive experiments with imagery and 3D models

KEY WORDS: 3D geovisualizations, user study, navigation, visuospatial memory, memory decline

\begin{abstract}
:
The use of virtual environments (VE) for navigation-related studies, such as spatial cognition and path retrieval has been widely adopted in cognitive psychology and related fields. What motivates the use of VEs for such studies is that, as opposed to real-world, we can control for the confounding variables in simulated VEs. When simulating a geographic environment as a virtual world with the intention to train navigational memory in humans, an effective and efficient visual design is important to facilitate the amount of recall. However, it is not yet clear what amount of information should be included in such visual designs intended to facilitate remembering: there can be too little or too much of it. Besides the amount of information or level of detail, the types of visual features ('elements' in a visual scene) that should be included in the representations to create memorable scenes and paths must be defined. We analyzed the literature in cognitive psychology, geovisualization and information visualization, and identified the key factors for studying and evaluating geovisualization designs for their function to support and strengthen human navigational memory. The key factors we identified are: i) the individual abilities and age of the users, ii) the level of realism (LOR) included in the representations and iii) the context in which the navigation is performed, thus specific tasks within a case scenario. Here we present a concise literature review and our conceptual development for follow-up experiments.
\end{abstract}

\section{INTRODUCTION}

Virtual environments have been widely used in psychological studies related to spatial cognition for simulating navigation and path retrieval tasks (Wiener \& Hanspeter, 2003, Meilinger et al., 2008), among others. By performing navigation experiments in virtual environments, researchers can control the unpredictable features and confounding situations occurring in the real world, and better isolate the factor(s) that may be causing the observed effects. Furthermore, from a visualization design point of view, it is valuable to identify the visual elements that may assist humans to perform better in navigation tasks. For example, an important question is, which visual elements best facilitate the retention of the visual information necessary for navigating effectively and efficiently? Does everyone remember the same visual elements, or does this differ based on certain cognitive and perceptual characteristics? In other words, when we design a geographic visualization with a specific purpose in mind, such as simulating navigation, we must keep the users of these visualizations in mind, along with the information we choose to represent in the visualization.

\section{STATE OF THE ART}

To answer questions such as the above, we first distinguish the main components that we are interested in studying. From a geographical perspective, we are motivated to develop geographical visualization designs that best facilitate information retention, and thus propose a visualization environment that is optimized to boost and train memory. Thus, the overarching goal in the study in relation to geographic visualization research is to derive geovisualization design principles for effective and efficient recall of visual and spatial elements on a path in navigation-related scenarios. Furthermore, taking a psychological perspective, we focus on the human, i.e., the user of the visualization. We thus define for whom the visualization will be designed, and for what task type(s) we can recommend the use of the proposed visualization environment given that the purpose is to boost and train the memory. These parameters (the stimuli, the participants, and the tasks/context) are standard experimental dimensions that should always be carefully considered when designing any controlled experiment (Martin, 2008; Çöltekin 2015).

In our context, the stimuli are the visualizations, and these are typically design products. Even the subtle choices in the way the visual information is presented may have a role in the later recall, i.e., basic visual variables such as color, size or position are important for memorability. Furthermore, some researchers argue that people better remember visual elements that are depicted in a manner similar to the real world; that consequently have recognizable features (e.g., Borkin et al., 2013). Supporting this idea, it has been suggested that virtual environments with high levels of realism (thus, including 'human recognizable' features) can be particularly useful for assisting human memory (Höffler, 2010; Mania and Chalmers, 2001).

In addition to the design choices, features types and level of realism in the visualization design, the technology itself also appears to play a role: Based on a literature survey, Loomis et

\footnotetext{
* Corresponding author
} 
al. (1999) suggested that the higher the visual quality of the virtual environment and the more the options of interaction and locomotion, the better the chances the environment is recalled. However, contradicting theories have also been proposed, such as the "naïve realism" and "naïve cartography" theories where the central argument is that people might prefer realistic and highly interactive visualizations, but do not necessarily perform better with them (Smallman and John, 2005; Hegarty et al., 2009). However, it is important to note that the latter two studies on "naïve realism" and "naïve cartography" focus on performance (e.g., accuracy and speed in completing the given tasks), and not explicitly on memory.

In a recent online choice experiment, it was demonstrated that for everyday route planning people preferred abstract 2D maps, and consulted photorealistic visualizations (such as satellite maps or 'street view' type representations) for identifying places of interest but not for navigation-related tasks in general (Çöltekin et al., 2015). Currently, a common understanding for most information visualizations is that abstraction (as opposed to realism) might be better for performance, because processing less information demands less from human cognitive resources and working memory capacity (Cowan, 2001). However, there is also evidence that participant background and abilities matter very much in such experiments, e.g., higher spatial abilities might benefit from 3D in learning and recall tasks while lowspatial ones do not (Huk, 2006), or expertise could affect success even in perceptual tasks (Bernabé Poveda and Çöltekin, 2014), as they may have developed strategies for working with spatial displays over time.

Similarly, the user's background is also important for the memorability of a visualization. In a navigation context (to recall paths one has taken), as in performance or perception studies, individual differences such as spatial abilities, age, expertise, may have an impact on how much people will be able to remember. Most importantly, psychological behavioral studies highlight the change occurring on memory capacity over the lifespan, offering ample evidence that there is a significant decline in our memory capacity as we age (Park et al., 1996). Thus, in a study such as we describe here, age is an important factor and should be considered for recruiting participants, i.e., different age groups may have different recall rates and potentially remember different visual elements. In fact, age appears to be important in performance and preferences of participants even if the focus of the study is performance and not memory (Schnürer et al., 2015). Additionally, spatial abilities have also been previously shown to affect performance as well as memory, especially in spatial tasks (Miyake et al., 2001). These changes regarding participant characteristics may increase the variation in the results in relation to different types of stimuli and thus should be carefully counterbalanced. For example, a more detailed, highly-realistic representation may lead to cognitive overload for low-spatial users, while an abstract representation may be easier to recall for high-spatial users who do not rely on visual memory as heavily, because they are possibly also able to use spatial memory.

The necessity to define representative task types (and eventually exact tasks) also naturally emerges while designing an experiment, because the scenarios in which we test the stimuli are critical for the generalizability of the findings. As stated earlier, when studying navigation in virtual environments, memory plays an important role (Montello et al., 2004). However, there are different memory processes and specifying the exact memory type relevant to the specific context (e.g., visual- and spatial-memory are highly relevant in navigation), and designing tasks that are in fact processed by these memory types is necessary for the validity of the results.

\section{BROAD CONCEPT AND FUTURE WORK}

A combination of the three key factors as introduced above can provide a baseline structure for developing effective and efficient design-guidelines for visualizations to boost and train memory (Fig. 1).

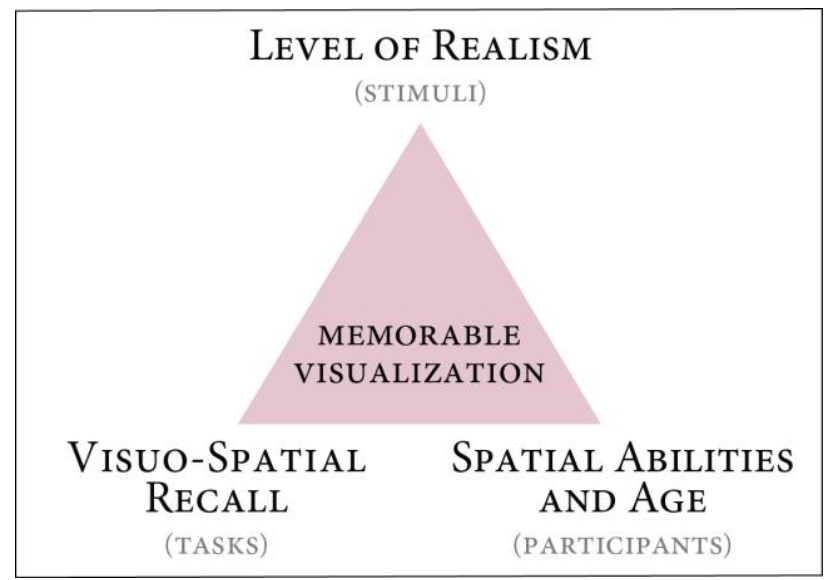

Figure 1. The broad conceptual design for experiments for testing the memorability of geovisualizations in our project.

The main contribution of this paper is a concise analysis of the literature connecting the memorability of 3D visualizations (specifically virtual reality representations) for different age and spatial ability groups. We plan to perform a set of empirical user studies to test our hypotheses generated according to this literature. With these empirical user studies, we aim to understand how people within different age groups retrieve information provided by different visualization designs, while we manipulate the level of realism in the visualizations. To set the baseline, we will include an abstract 3D visualization with no color and photo-texture, to diminish the recall of photorealistic visual information, thus the participants should rely mainly on spatial memory. A highly realistic 3D visualization with color and photo-texture will be on the other side of the spectrum. When using the highly realistic visualization, we expect participants to use both visual and spatial memory. We optimize a third design, balancing between abstraction and realism, in which we selectively include photorealistic elements in the visualizations which should help boost the participants' memory.

We select these photorealistic visual elements carefully and purposefully in terms of their content, position and frequency to specifically serve as memory-enhancing landmarks. Specifically, we select the location of the highlighted regions according to the theory of structural salience of landmarks (Röser et al., 2012). This theory suggests as the most prominent location of a landmark to be the structure on the intersection and towards where a turn needs to be made. Furthermore, we analyze the content of the photographic textures using saliency approaches and select 'human recognizable' elements which the participants can name in varying degrees. We hope to identify and clarify the strengths and weaknesses of each visualization, and how each of them interacts with age as well as individual spatial and visual abilities of the users. As an overarching goal, 
we intend to identify design guidelines and recommendations for age and spatial ability groups specifically to aid and improve memory capacity in the context of navigational path learning with simulated environments.

\section{ACKNOWLEDGEMENTS}

The project aims at improving the human performance with three-dimensional geographic visualizations and is funded by the Swiss National Science Foundation (SNSF project VISDOM, award number 200021_149670).

\section{REFERENCES}

Bernabé Poveda, M. A., \& Çöltekin, A. (2014). Prevalence of the terrain reversal effect in satellite imagery. International Journal of Digital Earth, 1-24.

Borkin, M., Vo, A., Bylinskii, Z., Isola, P., Sunkavalli, S., Oliva, A. and Pfister, H., 2013. What makes a visualization memorable?. Visualization and Computer Graphics, IEEE Transactions on, 19(12), pp.23062315.

Cowan, N. (2001). The magical number 4 in short-term memory: a reconsideration of mental storage capacity. The Behavioral and Brain Sciences, 24(1), 87-114; discussion 114-85.

Çöltekin, A. (2015). Mix well before use: Understanding the key ingredients of user studies. In ICC2015 Workshop on "Envisioning the Future of Cartographic Research." Curitiba, Brazil.

Çöltekin, A., Lokka, I.-E., \& Boer, A. (2015). The Utilization of Publicly Available Map Types by Non-experts -- A Choice Experiment. In Proceedings of the 27th International Cartographic Conference (ICC2015).

Hegarty, M., Smallman, H. S., Stull, A. T., \& Canham, M. S. (2009). Naïve Cartography: How Intuitions about Display Configuration Can Hurt Performance. Cartographica: The International Journal for Geographic Information and Geovisualization, 44(3), 171-186.

Höffler, T.N., 2010. Spatial ability: Its influence on learning with visualizations - a meta-analytic review. Educational psychology review, 22(3), pp.245-269.

Huk, T., 2006. Who benefits from learning with 3D models? The case of spatial ability. Journal of Computer Assisted Learning, 22(6), pp.392-404.

Loomis, J.M., Blascovich, J.J. and Beall, A.C., 1999. Immersive virtual environment technology as a basic research tool in psychology. Behavior Research Methods, Instruments, \& Computers, 31(4), pp.557-564.

Mania, K. and Chalmers, A., 2001. The effects of levels of immersion on memory and presence in virtual environments: A reality centered approach. CyberPsychology \& Behavior, 4(2), pp.247-264.

Martin, D. (2008). Doing Psychology Experiments. 2nd ed. Cengage Learning.

Meilinger, T., Knauff, M. and Bülthoff, H.H., 2008. Working memory in wayfinding - A dual task experiment in a virtual city. Cognitive Science, 32(4), pp.755-770.

Miyake, A., Friedman, N.P., Rettinger, D.A., Shah, P. and Hegarty, M., 2001. How are visuospatial working memory, executive functioning, and spatial abilities related? A latent-variable analysis. Journal of experimental psychology: General, 130(4), p.621.

Montello, D.R., Waller, D., Hegarty, M. and Richardson, A.E., 2004. Spatial memory of real environments, virtual environments, and maps. Human spatial memory: Remembering where, pp.251-285.

Park, D.C., Smith, A.D., Lautenschlager, G., Earles, J.L., Frieske, D., Zwahr, M. and Gaines, C.L., 1996. Mediators of long-term memory performance across the life span. Psychology and aging, 11(4), p.621.

Röser, F., Hamburger, K., Krumnack, A. and Knauff, M., 2012. The structural salience of landmarks: results from an online study and a virtual environment experiment. Journal of Spatial Science, 57(1), pp.37-50.

Smallman, H. S., \& John, M. S. (2005). Naive realism: misplaced faith in realistic displays. Ergonomics in Design: The Quarterly of Human Factors Applications, 13(3), 6-13.

Schnürer, R., Sieber, R., \& Çöltekin, A. (2015). The Next Generation of Atlas User Interfaces: A User Study with "Digital Natives." In Modern Trends in Cartography Lecture Notes in Geoinformation and Cartography (pp. 23-36).

Wiener, J.M. and Mallot, H.A., 2003. 'Fine-to-coarse' route planning and navigation in regionalized environments. Spatial cognition and computation, 3(4), pp.331-358. 